\title{
A JOINT DSO-TSO REACTIVE POWER MANAGEMENT FOR AN HV SYSTEM CONSIDERING MV SYSTEMS SUPPORT
}

\author{
Juliette MORIN \\ L2EP - France \\ juliette.morin@ensam.eu \\ Jean-Yves DIEULOT \\ CRIStAL - France \\ jean-yves.dieulot@polytech-lille.fr
}

\author{
Frédéric COLAS \\ L2EP - France \\ frederic.colas@ensam.eu
}

\author{
Xavier GUILLAD \\ L2EP - France \\ xavier.guillaud@ec-lille.fr
}

\begin{abstract}
This article deals with a joint DSO-TSO reactive power management to support TSO operations at HV level. This management is based on the coordination of several Volt Var Control schemes of MV networks able to control the reactive power at the DSO-TSO interface. The coordination is achieved through the resolution of an OPF problem. The definition of this latter is further discussed and recommendations raised.
\end{abstract}

\section{INTRODUCTION}

Distribution systems are undergoing major changes such as the insertion of Distributed Generation (DG), the replacement of overhead lines by underground cables or the evolution of the load. These changes have consequences on the Transmission System Operator (TSO) operations. In short, a decline and even reverse power flow is to be expected at the DSO-TSO interface [1], [2]. In France, this interface corresponds to the High Voltage (HV) / MV interface, typically $63 / 20 \mathrm{kV}$ or $90 / 20$ $\mathrm{kV}$. As a result, reverse reactive power flows can also be observed at EHV/HV system interface, especially at a low level of consumption. These power flows may exhibit a high variability correlated with the variability of weather dependent DGs production and loading conditions, and are hardly predictable. Also, the TSO can face difficulty to ensure a suitable regional reactive power provision or to mitigate the possible HV voltage rises. To deal with the aforementioned issues, new solutions for regional power balance can be found especially if large traditional power plant units are taken out of service to be replaced by renewable DGs mainly connected to Medium Voltage (MV) networks.

In the literature distribution systems are generally considered to support the TSO operations by adjusting the reactive power flows at their interface taking advantages of DGs reactive power capability. In the European Grid Code, Demand and Connection (DCC, [3]), new distribution systems are requested to have the technical capacity to restrain the reactive power flowing upwards the transmission system at low active power consumption, that is below $25 \%$ of their maximal power import capacity. In this DCC code, it is yet mentioned that other solutions may exist and can be considered; provided that both technical and economic benefits are jointly assessed by TSO and

\author{
Sébastien GRENARD \\ Enedis - France \\ sebastien.grenard@enedis.fr
}

DSO through common analysis.

This paper aims to propose a joint DSO-TSO coordination for reactive power management within a HV grid based on an Optimal Power Flow (OPF) technique. The objectives of the control are to maintain a suitable HV voltages profile while limiting the $\mathrm{EHV} / \mathrm{HV}$ reactive power exports. To this end, the control yields a reactive power target at each HV/MV interfaces. This target is then assumed to be considered by the real-time Volt Var Control (VVC) of MV grids that adjusts, among others, the reactive power references of distributed generation.

Part I discusses the relevance of such a control, Part II is dedicated to the definition of the control in terms of cost function and constraints. Simulations are performed on the medium scale system of Figure 1 that is a HV system encompassing several MV grids. In Part III, the limitations of the methods as well as recommendations to set up a joint DSO-TSO coordination are given.

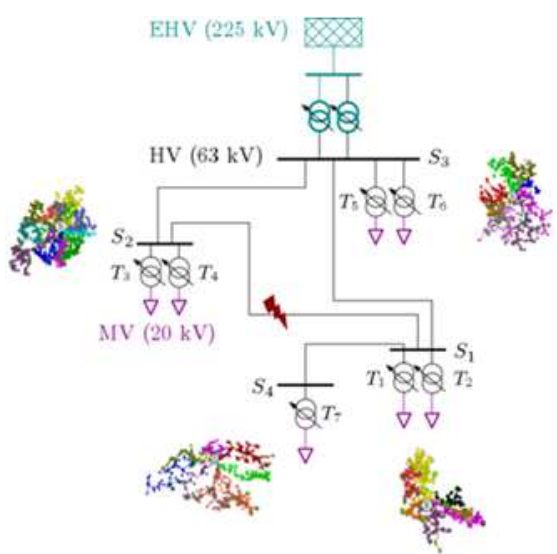

Figure 1: HV/MV medium scale system

\section{NEEDS AND REQUIREMENTS FOR A JOINT DSO-TSO REACTIVE POWER MANAGEMENT}

How distribution systems may support TSO operations through reactive power?

As a reminder, the $\mathrm{HV}$ voltage level in France is maintained by the means of EHV/HV On Load Tap Changer (OLTC) while the EVH level is managed through a classical three-level control. The influence of the reactive power from MV networks on the HV voltage is roughly proportional to the short circuit power of the substation $S_{c c}$. 


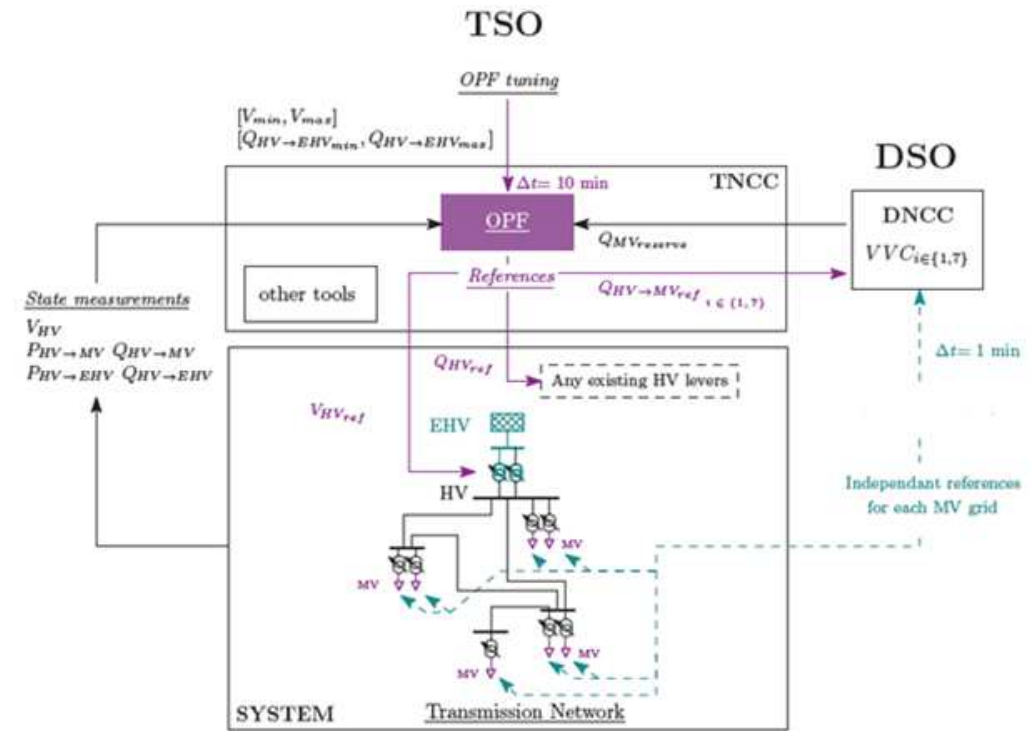

Figure 2: Schematic of the proposed OPF control and association with the DSO

Also, the sensitivity of the HV voltage of a HV/MV substation can be defined by the following equation (in per unit):

$$
\frac{\partial V}{\partial Q} \approx \frac{1}{S_{c c}}
$$

Taking a realistic example, if $S_{c c} \geq 500 \mathrm{MVA}$ then the sensitivity is below $0.002 \mathrm{pu} / \mathrm{MVAR}$. As a result, the reactive power support to maintain the corresponding voltage would turn out to be irrelevant. However considering weak transmission systems, typically rural ones, such a support could be worth considering.

Next, HV/MV substation short-circuit powers can be highly impacted by disturbances such as a line outage. In this specific case, a reactive power support can be helpful. Moreover, if several MV networks, connected within the $\mathrm{HV}$ region, exhibit reactive power exports upwards a HV grid, this reactive power can turn to be significant at the EHV/HV interface. These flows can then disturb the EHV voltage and reactive power control.

To sum up, a reactive power support is relevant for HV voltage support in case of weak HV system or for EHV operations purposes.

In the sequel, this support is assumed to be provided by distribution systems, taking advantage among others of the reactive power of DGs in the limit of their capacity and depending on the technology, size...

\section{$\underline{\text { Relevance of simulation on medium-scale systems }}$}

Generally, the topic of a reactive power support from the distribution systems is addressed from the transmission system point of view only. The fact that the reactive power of DGs is limited depending on the active power injection or the prevailing constraints (voltage, line thermal limits...) in distribution systems is often disregarded and the distribution grids modelled as constant or controllable loads. That is why there is a clear need to develop simulations combining both transmission and distribution systems as the system depicted in Figure 1, to take into account both TSO and DSO requirements and needs, and the true behavior of the system.

\section{Control assumptions and requirements}

To design a cost-effective and relevant support, there are several points to consider.

First, the reactive power limit that can be required from the MV grids is not constant but depends upon the prevailing conditions of the considered MV grid and of the HV voltage at the primary side of the HV/MV substation. Also, it will be assumed that the DSO is able to provide the MV networks reactive power reserves in real-time to the TSO. This reserve should be frequently updated by the Distribution Network Control Centre (DNCC) and sent to the (Transmission Network Control Centre) TNCC of the TSO. Thus, it is possible to ensure that the reactive power references yielded by the control will not jeopardize the DSO operations. Next, it also enables the TSO to anticipate if preventive or corrective actions should be taken to ensure the reliability and stability of the system.

Second, assuming this reactive power exists and is known, the DSO should be able to call for this reserve depending on the TSO needs and requirements. It is assumed that the distribution systems are equipped with a predictive controller, as the one presented in [4] inspired of [5] that controls both MV voltages and the reactive power exchange at the interface. In such a controller, the DSO voltage control requirements takes precedence over the reactive power targets at the HV/MV interface.

Third, it also implies that there exists a communication system between TSO and DSO as required in the European Grid Codes. In our work, it will be assumed that TSO and DSO are able to exchange relevant information regarding the reactive power capability and needs.

Last, the proposed control is assumed to be hosted within the TNCC as illustrated in Figure 2. The TSO plays a role of coordinator, and one of its assignments is to distribute 
the reactive power support among the distribution networks.

\section{DEFINITION OF AN OPF-BASED CONTROL FOR REACTIVE POWER MANAGEMENT}

\section{Suitability of OPF-based strategies}

Since there are cross-sensitivities between the HV/MV reactive power exchange of a HV/MV substation and the $\mathrm{HV}$ voltages of other substations, there are several alternative solutions to maintain a suitable HV voltage profile through a MV reactive power support. There exist also several strategies to reach a reactive power reference at the $\mathrm{EHV} / \mathrm{HV}$ system interface. As a result, using an OPF is of interest since this method enables to select the most appropriate solution that is an optimal solution with respect to a specific cost function. Next, OPF methods exhibit all advantages of optimization problem that are optimality, genericity and ability to deal with multiobjectives problems

\section{Presentation of the OPF strategy}

The goals of the control are first to enforce a suitable HV voltage profile while limiting the reactive power exchange at the EHV/HV interface. This second target must be consistent with the reactive power management and voltage control at the EVH level and is assumed to be set by the TSO. In this work, it is considered and assumed that the twofold objective (HV voltage and $\mathrm{EHV} / \mathrm{HV}$ reactive power) is necessary to ensure both reliable and safe operations of the power systems. Hence such goals are included in the optimization problem as hard constraints introduced hereafter. In other works, these goals should be enforced at any costs.

\section{Levers}

To this end, several levers $(L)$ can be considered within the HV system as illustrated in Figure 2 above: reactive power references $\left(Q_{H V}\right.$ ref $)$ of possible HV levers; voltage reference of the EHV/HV OLTC ( $\left.V_{\text {OLTC ref }}\right)$; reactive power references at each $\mathrm{HV} / \mathrm{MV}$ interfaces $\left(Q_{H V \rightarrow M V \text { ref }}\right) \ldots$ Here, the levers are reduced to the reactive power available from the MV grids: ( $Q_{H V \rightarrow M V \text { ref }}$ ). The TSO gives a reactive power reference for each MV grid (a reactive power target to meet at the HV/MV system interface). Then, each VVC hosted within the DNCC of the DSO acts independently from the others to enforce a suitable MV voltage profile, while trying to reach its own reactive power target assigned by the TSO.

\section{Cost function}

In order to reach coordination between the TSO and the $\mathrm{DSO}$, the following cost function to be minimized has been considered:

$$
J_{(\alpha)}=(1-\alpha)\left|Q_{H V \rightarrow M V \text { ref }}\right|+\alpha\left|P_{\text {losses }}\right|
$$

If any new levers are to be considered, their usage cost should be introduced in (2). Thus, the costs face by both TSO and DSO can be considered in the resolution of the

\section{OPF problem}

\section{Constraints}

The first constraints to be respected are the load flow equations that are not recalled herein.

The physical limitations of actuators $(L)$ should be considered and can be taken into account through the following equations to avoid inappropriate references:

$$
\begin{aligned}
& L_{\min } \leq L \leq L_{\max } \\
& L_{\min } \leq L \leq L_{\max }
\end{aligned}
$$

Then, the following constraints focusing on the control objectives should be considered:

$$
\begin{gathered}
V_{H V_{\text {min }}} \leq V \leq V_{H V_{\text {max }}} \\
Q_{H V \rightarrow E H V_{\text {min }}} \leq Q_{H V \rightarrow E H V} \leq Q_{H V \rightarrow E H V_{\text {max }}} \\
Q_{M V \rightarrow H V_{\text {min }}} \leq Q_{M V \rightarrow H V} \leq Q_{M V \rightarrow H V_{\text {max }}}
\end{gathered}
$$

In this paper, three different cases have been considered regarding constraints (6)-(7) as summarized in Table I below.

Table I : Sets of constraints to be studied

\begin{tabular}{|c|c|c|c|c|}
\hline \multirow{2}{*}{ Set } & \multicolumn{2}{|c|}{ Constraint (6) } & \multicolumn{2}{c|}{ Constraint (7) } \\
\cline { 2 - 5 } & $Q_{H V \rightarrow E H V_{\min }}$ & $Q_{H V \rightarrow E H V_{\max }}$ & $Q_{M V \rightarrow H V_{\min }}$ & $Q_{M V \rightarrow H V_{\max }}$ \\
\hline \hline$C_{V_{H V}}$ & $-\infty$ & $+\infty$ & $-\infty$ & $+\infty$ \\
\hline$C_{V_{H V}, Q_{H V}}$ & $-\infty$ & $+\infty$ & $-\infty$ & 0 \\
\hline$C_{V_{H V}, Q_{E H V}}$ & 0 & 0 & $-\infty$ & $+\infty$ \\
\hline
\end{tabular}

It should be noted that the set $C_{V_{H V}, Q_{E H V}}$ corresponds to one of the DCC requirement. Indeed according to this code, distribution systems must be able to prevent reactive power from flowing upwards when the consumption is below $25 \%$ of the maximum active power import. Also, by considering the aforementioned sets of constraints, it is possible to have a first insight regarding the needs in reactive power and depending on the cost function that has been selected.

\section{Modelling}

The model within the OPF control consists of:

- EHV/HV stations with OLTC transformers;

- HV lines and cables;

- HV/MV substations with OLTC transformers;

- Constant power loads that embody the MV grids, connected to the MV busbar of HV/MV substations;

- Constant power loads with adjustable reactive power that represents the reactive power available from the MV grids.

Since a constant load model is used to model the MV grids, this means that the active and reactive power consumed or produced by the MV grids are assumed to be independent from the OPF solutions.

\section{Resolution and constraints infeasibility}

It may happen that the constraints are too strict in 
particular if the MV reactive power reserve is insufficient, and thus cannot be met. Also, to ensure that the control still finds a solution limiting the constraints violation, a procedure based on the introduction of slack variables have been developed. Whenever needed, these slack variables are heavily penalized within the cost function.

\section{ILLUSTRATION OF THE OPF CONTROL PRINCIPLE ON A MEDIUM SCALE SYSTEM}

In order to illustrate the principle of the proposed control, several studies have been carried out in this work. More specifically, the evolution of the steady-state solution (level of losses and total amount of reactive power requested from MV grids) are studied depending on the weighting factor $\alpha$ of (2) and on the set of constraints to be enforced. To do so, static simulations have been performed considering only the OPF control, for a specific situation of the medium scale system that is at low consumption and high production. Furthermore, the reactive power reserve of each MV grid was assumed to be equal to 10 MVAR, which constitutes a significant reserve and enables to investigate a trend for the use of such a reserve. Since a specific HV system in a specific case have been considered, the results are not to be generalized but head in the direction of the need to define suitable DSO-TSO coordination and enable to draw recommendations for such a coordination.

\section{Case study}

The voltage, active and reactive powers at each MV bubsar of HV/MV substation are given in Table II, after the outage of the line $L_{S 2 \rightarrow S 1}$ since such a control is designed for system under emergency situation. It could be noted that except two transformers, there are reactive power flowing upward the HV systems, see Table II.

Table II : Voltage, active and reactive power at MV busbar of $\mathrm{HV} / \mathrm{MV}$ transformer after the line $\left(L_{S 2 \rightarrow S 1}\right)$ outage

\begin{tabular}{|c|c|c|l|l|}
\hline Transformer & $\begin{array}{c}V_{H V} \\
(\mathrm{pu})\end{array}$ & $\begin{array}{c}V_{M V} \\
(\mathrm{pu})\end{array}$ & $\begin{array}{c}P \\
(\mathrm{MW})\end{array}$ & $\begin{array}{c}Q \\
(\mathrm{MVAR})\end{array}$ \\
\hline \hline$T_{1}$ & 1.0744 & 1.0198 & -10.64 & $\mathbf{- 2 . 4 7}$ \\
\hline$T_{2}$ & 1.0744 & 1.0469 & -5.89 & $\mathbf{- 8 . 1 0}$ \\
\hline$T_{3}$ & 1.0174 & 1.0196 & -0.87 & 1.55 \\
\hline$T_{4}$ & 1.0174 & 1.0296 & -1.14 & $\mathbf{- 3 . 7 7}$ \\
\hline$T_{5}$ & 0.996 & 1.0195 & -0.85 & $\mathbf{- 3 . 2 3}$ \\
\hline$T_{6}$ & 0.996 & 1.0197 & -14.38 & 0.66 \\
\hline$T_{7}$ & 1.0921 & 1.0505 & 0.45 & $\mathbf{- 7 . 7 0}$ \\
\hline
\end{tabular}

Evolution of the steady state solution: influence of cost function and TSO requirements

Figure 3 gives the total amount of reactive power obtained for each simulation depending on the cost function that has been selected, while Figure 4 compares the level of active losses within the HV system.

From these figures, it can be observed that depending on the cost function and the selected constraints (e.g. TSO requirements), the solution yielded results in quite different level of loses and amount of reactive power requested. In short, the reactive power effort required from MV grids is immoderate when considering the losses minimization only ( $J_{\alpha=1}$ ) or when too hard constraints on the reactive power flows are required.

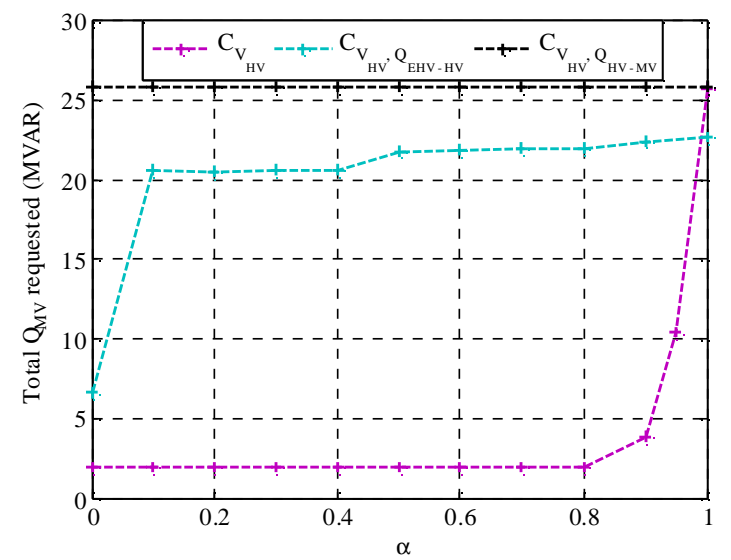

Figure 3: Total amount of reactive power depending on the OPF problem definition

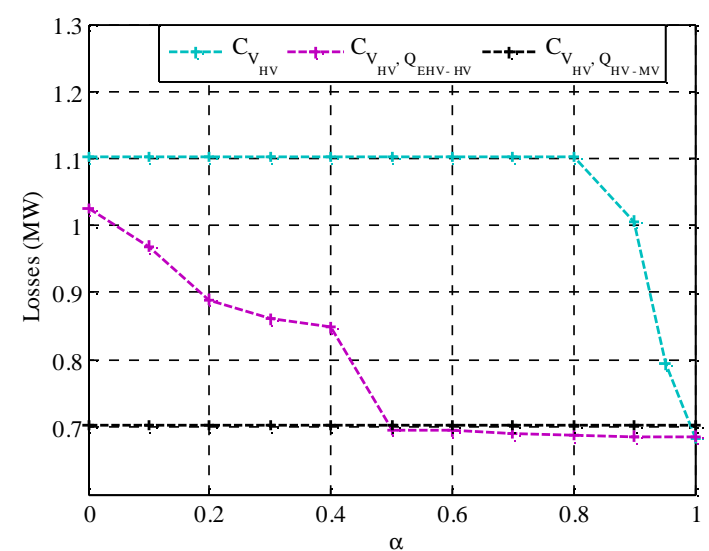

Figure 4 : Total amount of active power losses depending on the OPF definition

Reactive power reserve requirements

More specifically, according to Figure 3, the need in reactive power reserve is skyrocketing when tightening the constraints on the reactive power flows within the HV system. The DCC requirement (dark curve) is in this specific situation, the most "greedy" case regarding reactive power reserve. When considering $\alpha=1$, and without constraints on the HV reactive power flows (set of constraints $C_{V_{H V}}$ embodied by the violet curves) the needs of a MV reactive power support is quite increased. From Figure 3, there is a difference in the total amount of MV reactive power support of 27.6 MVAR between $J_{(\alpha \leq 0.8)}$ and $J_{(\alpha=1)}$. Hence, minimizing only the active losses while disregarding the cost of using the MV reactive power reserve is an irrelevant cost function.

Evolution of active losses

It should be noted that in any cases, the active losses after 
the corrective process are below the initial value of 1.18 MW (See Figure 4). Next, the more the reactive power is constrained, the less the reduction in active losses is possible. Indeed, there are no significant differences in the level of active losses reached with several value of $\alpha$ in the objective function $J_{(0 \leq \alpha \leq 1)}$ and either the set of constraints $C_{V_{H V}} ; C_{V_{H V}, Q_{H V}} ; C_{V_{H V}, Q_{E H V}}$

\section{Recommendations to set up an appropriate reactive power management}

Considering the first set of constraints $C_{V_{H V}}$ (where the constraints are only on $\mathrm{HV}$ voltages), the objectives of minimizing either the reactive power requested from MV grids and the HV system active losses are quite antagonistic. However, the differences are reduced especially for the second set of constraints $C_{V_{H V}, Q_{H V}}$. It is possible to reach a trade-off between the amount of reactive power requested and the level of losses within the $\mathrm{HV}$ system (See the results obtained with the cost function $\left.J_{(0 \leq \alpha \leq 1)}\right)$.

To sum up, in order to define an appropriate coordination between TSO and DSO, that implies to find a trade-off for the customer good, a sensitive and moderate effort should be requested from the MV grids, and the cost function should be negotiated considering every costs. From the results, the minimization of the active losses is not relevant especially if it is considered that the MV grid support is for safety and stability purposes. Also, the following cost function can be considered, with $\beta$ to be negotiated:

$$
J_{(\beta)}=(1-\beta)\left|Q_{H V \rightarrow M V \text { ref }}\right|+\beta\left(P_{\text {losses }}-P_{0}\right)
$$

Where $P_{\text {losses }}$ is a specific active losses level that should not be overcome insofar as possible.

The previous results have been obtained while considering the steady-state solutions in open-loop. The behavior and requirements of MV grids should be taken into account as well. Indeed, since the MV grids are equipped with VVC, the constraints are not instantaneously met, and the evolution of the HV/MV reactive power exchanges is also correlated with the prevailing constraints within the MV grids. For instance, it may happen that a specific MV grid cannot meet the reactive power demand at its HV/MV interface, and hence, the TSO may need to find an alternative solution.

\section{CONCLUSION}

To sum up, in this paper a coordination of several MV systems have been proposed in order to support the TSO operations. This coordination enables the TSO to send reactive power references at each $\mathrm{HV} / \mathrm{MV}$ interface in order to control the $\mathrm{HV}$ voltages and the $\mathrm{EHV} / \mathrm{HV}$ reactive power exchange. This paper explains that such a supervision is relevant for weak HV networks or for EHV reactive power purposes.

The MV distribution systems have been assumed to be equipped with real-time VVC allowing to control their own MV voltages as well as the reactive power at the HV/MV interface. The voltage control takes precedence. By studying a coordination based on the resolution of an $\mathrm{OPF}$, several conclusions have been raised.

Since the simulations have been carried out on a specific system, under specific conditions, the results are not to be generalized but enable to raise recommendations to set up a relevant and appropriate real-time DSO-TSO coordination:

- The constraints on reactive power flows may require a significant reactive power support from MV grids. Network planning studies should be jointly performed by TSO and DSO to ensure that the constraints are relevant and achievable;

- The management of reactive power should result from coordination between TSO and DSO; more specifically the cost function should be co-defined to find a cost-effective solution;

- The reactive power reserve should be accurately known and shared by the DSO to the TSO to prevent lack of reactive power support and avoid constraints infeasibility.

Future works will focus on dynamic simulations on the medium scale system to study the combination of the OPF supervisory algorithm and the VVCs of MV grids.

\section{REFERENCES}

[1] J. Morin, F. Colas, X. Guillaud and S. Grenard, 2015, "Determination and origins of reactive power flows in HV/MV substations", in Proc. 23 $3^{\text {rd }}$ CIRED conference, no 0414.

[2] ENTSO-E, ACER, 2016, "Network Code on Demand Connection" [Online] http://eurlex.europa.eu/legalcontent/EN/TXT/?uri=uriserv:OJ.L_.2016.223.01.0 010.01.ENG\&toc=OJ:L:2016:223:TOC

[3] C. Kaloudas and B. Marshall, 2014, "Initial Assexssmnet of Reactive Power Exchange at UK Grid Supply Point", International Conference and Exhibition on Electricity Distribution (CIRED) Workshop 2014, no. 0177.

[4] J.Morin, F.Colas, S. Grenard, J-Y. Dieulot, X. Guillaud, 2016, "Coordinated predictive control in active distribution networks with HV/MV reactive power constraint", in Proc. IEEE PES Innovative Smart Grid Technologies (ISGT) Europe.

[5] T.Van Cutsem and G. Valverde, Coordinated voltage control of distribution networks hosting dispersed generation, 2013, in Proc. $22^{\text {nd }}$ CIRED conference, no 1021 . 